\title{
The perceived psychological stressors and coping behaviours in university students, on a pre-registration programme.
}

\begin{abstract}
Purpose

The purpose was to investigate perceived stressors and coping behaviours in student nurses on a pre-registration programme of study. Stress in student nurses has been identified with decreased emotional well-being and poor academic achievement. The significance of stress and coping behaviours in students during training has implications for education and practice.
\end{abstract}

Design/methodology/approach

The present study recruited eighty-seven pre-registration student nurses in a cross-sectional design. Bivariate and multivariate analyses assessed the differences in field and year of study and the students' perceived stress and coping behaviours.

Findings

The findings showed that stress is a significant issue in nurse training. Fifty-three percent of the participants had levels higher than the mean. Interestingly, the present study found that high perceived stress was associated with avoidance behaviours. The most common type of perceived stress and ranked by highest factor were from written assignments and a lack of professional skills and knowledge. Their peer group and everyday life activities were shown as potential ways of coping with stressors. Thus, it seems reasonable to focus support on decreasing avoidant and enhancing stress-reducing behaviours.

Practical implications

Psychological stress and coping behaviours must be considered together as perceived stress is bound by the ability to ameliorate stress by managing helpful and unhelpful 
behaviours.

\section{Originality/value}

The findings may suggest that a potential benefit could come from the provision of helpful strategies such as peer group support and reduction of avoidant behaviours. Also, there seems to be a need for greater mental health literacy in dealing with stress during training.

\section{Keywords}

Student mental health; coping behaviours; perceived stress; mental health literacy; workforce issues

\section{Introduction}

The significance of stress and coping in pre-registration students has implications for academics and health professionals (Mitchell, 2018). The literature on nurse training has recognised that education, by its nature, is stressful because of the exposure to a range of potentially stressful situations. Some stressors that have been identified include dual aspects of training which include practice and academic components, types of emotional interaction with other professionals, interpersonal aspects of nursing care and dealing with abusive patients (Oner Altiok \& Ustun, 2013; Halter et al., 2017 and NHS England 2019). These potential stressors in students have significant consequences for both the individual and the education provider, such as psychological distress, poor academic performance and impaired professional practice (Heinen et al., 2013 and Health Education England, 2019). Effective coping behaviours can help students limit the effects of stressors but are dependent on the type of coping behaviours employed. Previous literature mainly focuses on stressors rather than coping behaviours. The studies that exist identify helpful coping behaviours such as seeking support from mentors, having hobbies and problem-focused resolution, and unhelpful behaviours which include social withdrawal and alcohol abuse (Han, 2015). 
Before proceeding to examine coping strategies, it is important to define what is meant by distress. Emotional stress is experienced when high perceived stress and poor compensatory coping behaviours are present (Briggs et al., 2017). This is sometimes called distress. This distress can sometimes be long term if the trigger in the environment persists or coping behaviours are unable to diminish the stress. Long term exposure to stress can cause physical and psychological health issues. Physical symptoms include increased heart rate, elevated blood pressure and sleep disturbance (Salvagioni et al., 2017). Psychological symptoms include anxiety, depression and somatic illness such as headaches (Salvagioni et al., 2017). High levels of psychological distress can be associated with behaviours, such as alcohol or drug abuse (Briggs et al., 2017).

Having defined what is meant by distress, it is necessary to discuss what is meant by coping strategies. Individuals can employ inward or outward coping behaviours. Inward strategies could include acceptance and reframing (Darabi, Macaskill \& Reidy, 2017). Outward tactics include activation strategies such as pleasure and attainment (Mitchell, 2019). Coping strategies can be described as either active; dealing with actual events, or reactive; dealing with the perceived or imagined stressor via internal thoughts and feelings. Coping behaviours, in nurses, can be divided into four broad categories: problem-solving, avoidance, optimism and transference (Sheu et al., 2002).

A more detailed account of coping strategies is given utilising a cognitive behavioural approach. The cognitive behavioural model of the impact of an event on the individuals' psychological or physical well-being, is taken as the balance of compensatory actions and cognitions to adapt to the environmental demands or towards the perceived stressor (Schroder et al., 2017). The main two types of coping: problem-focused, which tries to change the source of the stressor and emotion-focused, which works to improve the feelings or emotions accompanying the stressor. Problem-focused strategies include actions such as 
practising a speech before giving a presentation or preparing several key slides for the session. However, emotion-focused strategies include relaxation techniques and other enjoyable activities aimed at reducing the emotional stress connected with the stressor.

The previous section has so far defined what is meant by the main two types of coping, and it is now necessary to discuss three other important strategies. These include transference, optimism and avoidance. The psychodynamic view of transference is often used to describe a redirection of feelings from the source or object of the stress to a new unrelated source or object (Diamond, 2016). This might involve transferring the stress and associated pain from the workplace onto a non-threating environment such as the home or family environment. Optimism is a positive and emotional focused strategy where an individual has the strength of mind to maintain a belief that everything will work out, or they will succeed in overcoming the issue. Avoidance is a negative emotion-focused strategy and involves denial of the stressor and can involve escapism through alcohol or substance misuse.

Turning now to the specific literature on stress in the nursing profession. A systematic review of stress in nurses found that stress was an important issue for student nurses and the profession (Halter et al., 2017). This, combined with a recent survey by NHS England (2019) found that nearly $40 \%$ of nurses felt unwell due to work-related stress in practice in the previous 12 months. Another significant aspect of stress in student nurses is the transition stresses on starting a nurse training course, entering each clinical placement and on starting their first post as a registered nurse (Health Education England 2019). To conclude this section, the literature identifies that stress is a significant issue for nurses, with key transition stages being identified as stressful.

The present study could prompt the development of new strategies to reduce stress on students and to identify possible support strategies. Stress reduction in students may improve student well-being and performance, reduce the high attrition rates, and retain 
nurses in training (NHS Improvement, 2018 and Buchan, Charlesworth, Gershlick, Seccombe, 2019). This is at a time when student retention is paramount to the health service workforce (Health Education England, 2018; Buchan, et. al., 2019). Furthermore, findings could strengthen the need for structured support in student nurse training.

This study has the potential to inform our understanding of perceived stressors and coping behaviours in pre-registration nurses.

The aims of the present study are:

1. Does coping vary with demographics characteristics such as year and field of study?

2. Does perceived stress vary with demographics such as year and field of study?

3. Is there a relationship between perceived stress and types of behavioural coping?

\section{Methods}

Study design

The study was cross-sectional in its design. Bivariate and multivariate analyses assessed the differences in field and year of study and the students' perceived stress and coping behaviours.

\section{Participants}

A total of 87 pre-registration nurses in the North West of England ( $8 \%$ male and $92 \%$ female; mean age 27 years, SD 7.3, range 18-51) were recruited for the study. A priori sample size for the difference between two independent means (Faul et al., 2007) within two groups, power of 0.80 with medium effect size $(f=0.6)$ calculated the desired sample size of 82 . Similarly, a priori sample size for correlation of means (Faul et al., 2007) with a power of 0.80 and medium effect size $(f=0.3)$ indicated a similar desired sample size. 
Measures

Perceived Stress Scale (PSS)

The PSS (Sheu et al., 1997) consists of questions designed to reflect a respondent's perceived stressors in training. The scale consists of 29 Likert items, ranging from 0 ("Never") to 4 ("Always"). The items fall into six perceived stress categories: nursing care of patients (8 items), academics and clinical staff ( 6 items), from workload and assignments (5 items), peer group / everyday life (4 items), low level of knowledge \& skills (3 items) the clinical environment (3 items). High summed scores indicate higher perceived stress. The total summed scores can range from 0-116. Cronbach's alpha for this sample was 0.94 .

\section{Coping Behaviour Inventory (CBI)}

The CBI (Sheu et al., 2002) consists of questions designed to reflect nursing students' coping behaviours. The scale consists of 19 items, ranging from 0 ("Never") to 4 ("Always"). The items fall into four coping behaviour categories: avoidance (6 items), problem-solving (6 items), optimistic (4 items) and transference (3 items). High scores indicate higher use of the associated coping behaviour. Cronbach's alpha for this sample was 0.82 .

\section{Procedure}

Ethical permission was granted from the Faculty Ethics Committee for Health and Social Care. Participation in the study was not only voluntary but also anonymous. Recruitment was undertaken via an e-mail distribution list. To be included as a participant, students had to be undertaking one of the four pre-registration nursing fields and be registered for full-time study. Before completing an online questionnaire, students were asked to indicate consent to participate after reading a participant information sheet.

The PSS and CBI scores were determined from the individual items according to the instructions on each scale. The item scores of each subscale were calculated to give six sub-scale scores for the PSS and four subscale scores for the CBI. Percentages were 
computed for those participants whose perceived stress scores were above the mean. The items for both PSS and CBI were ranked by calculated mean scores.

\section{Statistical methods}

Statistical analysis involved correlational and one-way ANOVA's using IBM SPSS (Statistics for Windows, Version 24.0). In addition, descriptive statistics, appropriate to the level of measurement, were used to report the study variables. No missing data due to the design of online form. Checks for normality were undertaken, and assumptions of each statistical analysis were observed. A significance of $p<.05$ was adopted.

\section{Results}

\section{Levels of perceived stress and coping behaviour}

The mean of stress perceived by student nurses was $1.84(S D=.68)$. Results showed that $52.9 \%$ of pre-registration nurses had stress levels above the mean. The most commonly occurring type of perceived stress and ranked by the highest factor, and above the mean, were related to stress from written assignments and overall workload $(M=2.41, S D=.96)$ and stress from a lack of professional skills and knowledge $(M=2.06, S D=.98)$. The result of the factor ranking for the perceived stress scale is presented in table 1.

The mean of all coping strategies among students was $1.88(S D=.48)$. The most common type of coping strategies utilized and ranked by highest factor, and above the mean, were problem solving $(\mathrm{M}=2.65, \mathrm{SD}=.82)$, stay optimistic $(\mathrm{M}=2.32, \mathrm{SD}=.70)$ and transference $(M=2.18, S D=.89)$. The result of the factor ranking for the coping behaviour inventory is presented in table 1.

Relationship between types of coping and perceived stress The correlation between the mean score on the Perceived Stress Scale (PSS) and Coping Behaviour Inventory $(\mathrm{CBI})$ was $r(87)=.39, p<.001$. Hence, the correlation is moderate and 
significant (Evans, 1996). Pearson correlational test was also utilised to examine the correlation between factors on the perceived stress and coping behaviours scales, showing a significant relationship between overall perceived stress and avoidance coping subscale ( $r$ $=.63, p=<.001)$. Hence, the correlation is strong and significant (Evans, 1996). The result of the correlation between overall PSS and CBI and subscales is presented in table 2.

There was a significant relationship between avoidance and stressors 'from taking care of patients' $(r=.43, p<.001)$, 'from academic and clinical staff' $(r=.54, p<.001)$, 'written assignments and overall workload' $(r=.50, p<.001)$, 'from peer group / everyday life' $(r=$ $.52, p<.001)$, 'from a lack of professional skills and knowledge' $(r=.40, p<.001)$, 'from the clinical environment' $(r=.44, p<.001)$. This provides evidence that pre-registration nurses who perceived high levels of stress overly used avoidance behaviours as the main way to ameliorate the effects of stress. There were no other significant correlations between total PSS and subscales score with problem-solving, staying optimistic or transference.

Differences in perceived stress and coping behaviours with year and field of study It was hypothesized $(\mathrm{H} 1)$ that there would be a difference in the Perceived Stress Scale (PSS) and Coping Behaviour Inventory (CBI) scores with year and field of study. One-way ANOVA was utilized to assess the impact of the year of study on perceived stress. It can be seen (see table 3) that year 2 group has the smallest mathematical mean level of student perceived stress $(M=1.79, S D=.78)$ and year 3 group was associated with the numerically highest mean level of perceived stress $(M=1.87, S D=.60)$. Prior to the analysis, the assumptions of normality were evaluated, skew $<2.0$ and kurtosis $<9.0$; Schmider et al., 2010 see Table 3). The ANOVA yielded a non-significant effect, $F(2,84)=.11, p=.89, \eta^{2}=$ .003. Therefore, the null hypothesis $\left(\mathrm{H}_{0}\right)$ of no difference between the means was accepted. 
Similarly, a one-way ANOVA was utilized to assess the impact of the year of study on coping behaviour. It can be seen (see table 3) that year 1 group has the smallest mathematical level of coping behaviour $(\mathrm{M}=1.77, \mathrm{SD}=.61)$ and year 2 group has the highest mathematical mean level of coping behaviour $(\mathrm{M}=2.04, \mathrm{SD}=.39)$. The independent between groups ANOVA yielded a non-significant effect, $F(2,84)=2.65, p=.08, \eta 2=.059$. Thus, the null hypothesis $\left(\mathrm{H}_{0}\right)$ of no difference was accepted.

The descriptive statistics on perceived stress across the four pre-registration fields are reported in table 3. It can be seen that the pre-registration (child) was associated with the smallest mathematical mean level of perceived stress $(M=1.60, S D=.37)$ and the preregistration (learning disabilities) was associated with the highest mathematical mean level of perceived stress $(M=2.08, S D=.71)$. An ANOVA was conducted in order to test the hypothesis that pre-registration field of study had an effect on perceived stress. The ANOVA yielded a non-significant effect, $F(3,83)=.65, p=.58, \eta 2=.023$. Thus, the null hypothesis $(\mathrm{H} 0)$ of no difference was accepted.

The descriptive statistics on coping behaviour across the four pre-registration fields are reported in table 3. It can be seen that pre-registration (child) was associated with the smallest mathematical mean level of coping behaviour $(M=0.97, S D=1.38)$ and the preregistration (learning disabilities) was associated with the highest mathematical mean level of coping behaviour $(\mathrm{M}=2.12, \mathrm{SD}=.45)$. An ANOVA was conducted in order to test the hypothesis that field of study had an effect on coping behaviour. The Levene's $F$ test, $F(3$, $83)=3.69, p=.02$ was significant, but the assumptions for homogeneity of variance was not met.

\section{Predictors of perceived stress and coping behaviours}

Due to the lack of significant findings with demographics characteristics of study year and field of study, attention was focused on the relationship between perceived stress and 
coping as the present study has shown a correlation between perceived stress and avoidant coping. Therefore, further analysis was undertaken to ascertain the predictors of perceived stress and behaviours associated with coping. One of two multiple linear regressions were conducted. The four categories of coping behaviours were entered for the first analysis as independent variables (Table 4). One significant independent variable of perceived stress was found; avoidant behaviour accounted for $35.8 \%$ of the total variance of the perceived stress attributable to that variable alone. The six categories of perceived stressors were entered for the second analysis as independent variables. One significant independent variable of coping was found; peer group / everyday life accounted for $14.4 \%$ of the total variance of coping attributable to that variable alone.

\section{Discussion}

The main aim was to gain an understanding of the level of perceived stress and behaviours associated with coping in pre-registration nurses. This study found that problem solving, and optimism were the two main strategies used to cope with stressors, while transference and avoidance were the least used strategies, these are congruent with previous studies (AlZayyat \& Al-Gamal, 2014). Furthermore, this study shows high levels of stress when using avoidance behaviours. The most common type of perceived stressors and ranked by highest factor were related to stress from written assignments and workload. The findings are equivalent to other researchers (Labrague et al., 2018). In the present study, the second common stressor was a low level of professional knowledge and skills in the clinical environment. Stress-related to a low level of knowledge in practice was the main source reported by students (Jimenez et al., 2010). A key implication is that there is a need for teaching techniques in dealing with stressors in the training curriculum.

Studies have found that approximately $48 \%$ of student nurses had levels of stress above the mean (Khater et al., 2014). This is comparable with nearly $53 \%$ of the student with stress 
levels above the mean in the present study. These percentages are comparable, given the same measurement tool was used in both studies. It is acknowledged that high levels of perceived stress can affect attention and concentration in students (Horgan et al., 2016). High stress may lead to poorer academic achievement and low levels of retention (Labrague et al., 2018).

The finding that written assignments and overall workload show the highest source of stress is reported in previous studies (Hamaideh et al., 2017 and Labrague et al., 2018). The most common types of stressors and ranked by individual items in the scale were worrying about bad grades and low level of knowledge in practice. Previous studies show that preregistration students are vulnerable to stress from both clinical and academic components (Hamaideh et al., 2017 and Jamshidi et al., 2016).

Of related importance, is how students cope with stressors, as this will relate to how the individual will experience the impact on their emotional well-being. Coping strategies can act to buffer stressors and reduce the perceived stressfulness of the event (Schroder et al., 2017). Psychological stress is experienced when, either, stress is perceived to threaten goals and / or maladaptive strategies are used to reduce stressors. The present study found that the two most common types of coping behaviour utilized and ranked by highest factor were problem-solving and optimism and the least common ranked by lowest factor were transference and avoidance strategies. Several studies confirm that the most common coping behaviour is problem-solving (Al-Zayyat \& Al-Gamal, 2014 and Chen \& Hung, 2014) and the least common is avoidance (Hamaideh et al., 2017 and Kim \& Han, 2015).

Avoidance was ranked behind other coping strategies, but a significant relationship between total perceived stress and avoidance was observed. The present study found that participants who showed higher indexes of stress also showed higher indexes of avoidance on each of the six subscales. Avoidance is generally recognized as only a temporary coping 
strategy (Fornes-Vives et al., 2016; Hamaideh et al., 2017 and Kim \& Han, 2015) and not a long-term strategy to address underlying stressors. Avoidance typically involves physical or psychological withdrawal, such as avoiding tutors and mental distraction, such as the use of alcohol or drugs.

Furthermore, in this study, avoidance accounted for the highest total variance, as a predictive factor, within perceived stress. This finding may indicate the need for changes in the academic environment to promote supportive coping behaviours among pre-registration students. Additionally, on the CBI; peer group / everyday life showed the highest total variance for the most utilised adaptive strategies in reducing stress.

This study found that year and field had no significant effect on perceived stress and coping behaviours employed. The literature shows some inconsistency in findings with some showing an effect and others showing no effect for year or field of study. Previous studies have identified student's year of study as being a significant predictor of perceived stress and coping behaviours (Fornes-Vives et al., 2016 and Labrague et al., 2018). Both studies showed a positive change in perceived stress and coping strategies as the students progressed in their training. This is not surprising since core competencies and knowledge would be gained as they progress in their training and experience. However, there is no evidence for the field of study showing an effect on perceived stress or coping strategies in the present study. This might be somewhat counterintuitive as mental health literacy amongst mental health students might be thought to be greater in disciplines related to mental health knowledge (Marie et al., 2017 and Wickstead \& Furnham, 2017).

The present finding that moderate levels of stress were reported in half the sample suggests there may be a benefit in the incorporation of coping strategy literacy in pre-registration programmes. Previous findings support the importance of utilising strategies to reduce 
students' perception of stressors and facilitating adaptive coping strategies to deal with these (Al-Zayyat \& Al-Gamal, 2014). Initiatives such as Mental Health First Aid (Lipson et al., 2014) schemes help students to recognise stress and anxiety symptoms. Such actions promote mental health literacy and provide helpful advice on protective strategies (Wong et al., 2015). Academics should promote mental health literacy and mindfulness techniques among students (Galante et al., 2016). Such student support is deemed necessary and seen as a priority to promote well-being (Holt \& Powell, 2017 and Universities UK/MWBHE, 2015).

\section{Limitations}

The quantitative cross-sectional design has only allowed for statistical analysis of means rather than the rich and depth data that would have been gained from qualitative or mixedmethod designs. Quantitative and qualitative methods could be used together to mitigate the weaknesses of a just one methodological approach. Also, the observational design only allows for associations to be inferred rather than cause and effect of perceived stress and coping strategies amongst pre-registration nurses. Studies in the future could undertake a longitudinal design which would strengthen the understanding of the link between perceived stress and the use of differential coping behaviours. Future research strategies should look at outcomes; both academic and clinical in pre-registration students to fully ascertain the perceived stress and coping behaviours in nurses in training.

\section{Applications}

The present findings that students using problem-focused and optimism strategies, which are associated with experiencing less stress; rather than, an emotion-focused approach such as avoidance which was associated with increased stress, can lead to valuable practical advice. The findings may provide potential strategies that would lessen the impact of perceived stress and provide adaptive coping strategies. It would seem reasonable to suggest that problem-focused coping skills could be a useful recommendation to incorporate as a part of broader coping skills courses embedded within present nurse training curricula 
or as elective courses for enhancement. Individually, academic tutors can use tutorial support to build rapport with each of their students to help identify the most vulnerable and provide advice on strategies. This could be incorporated into focused discussions on personal stressors or situation that make them stressed and provide advice on strategies for that student. Interventions may include the provision of mindfulness-based sessions (Mak et al., 2017) and providing student well-being support within academic training programmes (Horgan et al., 2016). Furthermore, it seems pertinent to suggest that help could be provided to enhance beneficial strategies such as peer group support and to reduce avoidant behaviour. Thus, there is an opportunity to provide support and mental health literacy, in preregistration curricula, that may help with stressors during training.

\section{Conclusions}

This study compared perceived stressors and coping behaviours in pre-registration nurses and provided key behaviours that contribute to stress and behaviours that ameliorate the effect. Interestingly, the present study found that high perceived stress was associated with avoidance behaviours. Specifically, those students who showed high stress also showed higher indexes of avoidance. The peer group and everyday life activities were shown as a potentially positive way of coping with stressors. This study may provide valuable empirical findings for those researchers undertaking future research in this area.

\section{Acknowledgements}

The present research received support from an internal Quality Research $(Q R)$ fund. 


\section{References}

Al-Zayyat, A.S. and Al-Gamal, E. (2014), "Perceived stress and coping strategies among Jordanian nursing students during clinical practice in psychiatric/mental health courses", International Journal of Mental Health Nursing, Vol. 23 No. 4, pp. 326-335, doi: 10.1111/inm.12054.

Biggs, A., Brough, P. and Drummond, S. (2017), Lazarus and Folkman's Psychological Stress and Coping Theory, John Wiley \& Sons, Chichester.

Buchan, J. Charlesworth, A. Gershlick, B. and Seccombe, I. (2019), "A critical moment: NHS staffing, trends, retention and attrition", Health Foundation, London, available at: www.health.org.uk/publications/reports/a-critical-moment (accessed 20 March 2020).

Chen, Y.W. and Hung, C.H. (2014), "Predictors of Taiwanese baccalaureate nursing students' physio-psycho-social res responses during clinical practicum", Nurse Education Today, Vol. 34 No. 1, pp. 73-77, doi: 10.1016/j.nedt.2013.02.021.

Darabi, M., Macaskill, A. and Reidy, L. (2017), "Stress among UK academics: identifying who copes best", Journal of Further and Higher Education, Vol. 41 No. 3, pp. 393-412, doi: 10.1080/0309877X.2015.1117598.

Diamond, M.A. (2016), Discovering Organizational Identity: dynamics of Relational Attachment, University of MO Press, MO.

Evans, J.D. (1996), Straightforward Statistics for the Behavioral Sciences, Thomson Brooks/Cole Publishing, Pacific Grove, CA.

Faul, F., Erdfelder, E., Lang, A.G. and Buchner, A. (2007), "G*power 3: a flexible statistical power analysis program for the social, behavioral, and biomedical sciences", Behavior Research Methods, Vol. 39 No. 2, pp. 175-191, doi: 10.3758/BF03193146.

FornéS-Vives, J., Garcia-Banda, G., Frias-Navarro, D. and Rosales-Viladrich, G. (2016), "Coping, stress, and personality in Spanish nursing students: a longitudinal study", Nurse Education Today, Vol. 36, pp. 318-323, doi: 10.1016/j.nedt.2015.08.011.

Galante, J., Dufour, G., Benton, A., Howarth, E., Vainre, M., Croudace, T.J., Wagner, A.P., S tochl, J. and Jones, P.B. (2016), "Protocol for the mindful student study: a randomised controlled trial of the provision of a mindfulness intervention to support university students' well-being and resilience to stress", BMJ Open, Vol. 6 No. 11, p. e012300, doi: 10.1136/bmjopen-2016-012300.

Halter, M., Boiko, O., Pelone, F., Beighton, C., Harris, R., Gale, J., Gourlay, S. and Drennan, V. (2017), "The determinants and consequences of adult nursing staff turnover: a systematic review of systematic reviews", BMC Health Services Research, Vol. 17 No. 1, p. 824, available at: www.ncbi.nlm.nih.gov/pubmed/29246221 (accessed 24 March 2020).

Hamaideh, S.H., Al-Omari, H. and Al-Modallal, H. (2017), "Nursing students' perceived stress and coping behaviors in clinical training in Saudi Arabia", Journal of Mental Health, Vol. 26 No. 3, pp. 197-203, doi: 10.3109/09638237.2016.1139067. 
Han, M.R. (2015), "A study of emotional intelligence and coping strategies in baccalaureate nursing students", International Journal of Bio-Science and Bio-Technology, Vol. 7 No. 3, pp. 275-282.

Health Education England (2018), "RePAIR: reducing pre-registration attrition and improving retention", available at: hee.nhs.uk/our-work/reducing-pre-registration-attrition-improvingretention (accessed 20 March 2020).

Health Education England (2019), NHS Staff and Learners' Mental Wellbeing Commission, Health Education England.

Heinen, M.M., Van-

Achterberg, T., Schwendimann, R., Zander, B., Matthews, A., Kozka, M., Sjetne, I.S., Casba s, T.M., Ball, J. and Schoonhoven, L. (2013), "Nurses' intention to leave their profession: a cross sectional observational study in 10 European countries", International Journal of Nursing Studies, Vol. 50 No. 2, pp. 174-184, doi: 10.1016/j.jjnurstu.2012.09.019.

Holt, M. and Powell, S. (2017), "Healthy universities: a guiding framework for universities to examine the distinctive health needs of its own student population", Perspectives in Public Health, Vol. 137 No. 1, pp. 53-58, doi: 10.1177/1757913916659095.

Horgan, A., Sweeney, J., Behan, L. and McCarthy, G. (2016), “Depressive symptoms, college adjustment and peer support among undergraduate nursing and midwifery students", Journal of Advanced Nursing, Vol. 72 No. 12, pp. 3081-3092, doi: 10.1111/jan.13074.

Jamshidi, N., Molazem, Z., Sharif, F., Torabizadeh, C. and Kalyani, M.N. (2016), "The challenges of nursing students in the clinical learning environment: a qualitative study", The Scientific World Journal, Vol. 2016, p. 7, doi: 10.1155/2016/1846178.

Jimenez, C., Navia-Osorio, P.M. and Diaz, C.V. (2010), "Stress and health in novice and experienced nursing students", Journal of Advanced Nursing, Vol. 66 No. 2, pp. 442-455, doi: 10.1111/j.1365-2648.2009.05183.x.

Khater, W., Akhu-Zaheya, L. and Shaban, I. (2014), "Sources of stress and coping behaviours in clinical practice among baccalaureate nursing students", International Journal of Humanities and Social Science, Vol. 4 No. 6, pp. 194-202.

Kim, M.R. and Han, S.J. (2015), "Nursing students' emotional intelligence and coping strategies", Advanced Science and Technology Letters, Vol. 88, pp. 53-56.

Labrague, L.J., McEnroe-

Petitte, D.M., Papathanasiou, I.V., Edet, O.B., Tsaras, K., Leocadio, M.C., Colet, P., Kleisiari s, C.F., Fradelos, E.C., Rosales, R.A., Vera Santos-Lucas, K. and Velacaria, P.I.T. (2018), "Stress and coping strategies among nursing students: an international study", Journal of Mental Health, Vol. 27 No. 5, pp. 402-408, doi: 10.1080/09638237.2017.1417552.

Lipson, S.K., Speer, N., Brunwasser, S., Hahn, E. and Eisenberg, D. (2014), "Gatekeeper training and access to mental health care at universities and colleges", Journal of Adolescent Health, Vol. 55 No. 5, pp. 612-619, doi: 10.1016/j.jadohealth.2014.05.009.

Mak, W.W., Chio, F.H., Chan, A.T., Lui, W.W. and Wu, E.K. (2017), "The efficacy of internetbased mindfulness training and cognitive-behavioral training with telephone support in the 
enhancement of mental health among college students and young working adults: randomized controlled trial', Journal of Medical Internet Research, Vol. 19 No. 3, p. e84, doi: 10.2196/jmir.6737.

Marie, M., Hannigan, B. and Jones, A. (2017), "Resilience of nurses who work in community mental health workplaces in Palestine", International Journal of Mental Health Nursing, Vol. 26 No. 4, pp. 344-354, doi: 10.1111/inm.12229.

Mitchell, A.E.P. (2018), "Psychological distress in student nurses undertaking an educational programme with professional registration: their perceived barriers and facilitators in seeking psychological support', Journal of Psychiatric and Mental Health Nursing, Vol. 25 No. 4, pp. 258-269, doi: 10.1111/jpm.12459.

Mitchell, A.E.P. (2019), "Depression and behaviourism: beating 'the blues' with activity”, Psychology Review, Vol. 24 No. 3, pp. 2-4.

NHS England (2019), NHS Staff Survey 2018: National results briefing, available at: www.nhsstaffsurveys.com/Page/1064/Latest-Results/2017-Results (accessed 23 March 2020).

NHS Improvement (2018), "Staff retention support programme: one year on", NHS Improvement, available at: https://improvement.nhs.uk/resources/staff-retention-supportprogramme-one-year

Oner Altiok, H.O. and Ustun, B. (2013), "The stress sources of nursing students", Educational Sciences: Theory \& Practice, Vol. 13 No. 2, pp. 760-766.

Salvagioni, D.A., Melanda, F.N., Mesas, A.E., González, A.D., Gabani, F.L. and de Andrade, S.M. (2017), "Physical, psychological and occupational consequences of job burnout: a systematic review of prospective studies", PloS One, Vol. 12 No. 10, p. e0185781.

Schmider, E., Ziegler, M., Danay, E., Beyer, L. and Bühner, M. (2010), "Is it really robust? Reinvestigating the robustness of ANOVA against violations of the normal distribution assumption", Methodology, Vol. 6 No. 4, pp. 147-151, doi: 10.1027/1614-2241/a000016.

Schroder, H.S., Yalch, M.M., Dawood, S., Callahan, C.P., Donnellan, M.B. and Moser, J.S. ( 2017), "Growth mindset of anxiety buffers the link between stressful life events and psychological distress and coping strategies", Personality and Individual Differences, Vol. 110, pp. 23-26, doi: 10.1016/j.paid.2017.01.016.

Sheu, S., Lin, H.S. and Hwang, S.L. (2002), "Perceived stress and physio-psycho-social status of nursing students during their initial period of clinical practice: the effect of coping behaviors", International Journal of Nursing Studies, Vol. 39 No. 2, pp. 165-175, doi: 10.1016/S0020-7489(01)00016-5.

Sheu, S., Lin, H.S., Hwang, S.L., Yu, P.J., Hu, W.Y. and Lou, M.F. (1997), “The development and testing of perceived stress scale of clinical practice", Nursing Research, Vol. 5 No. 4, pp. 341-351.

Universities UK/MWBHE (2015), "Student mental wellbeing in higher education: good practice guide", UUK, London, available 
at: www.universitiesuk.ac.uk/highereducation/Documents/2015/StudentMentalWellbeinglnH E.pdf

Wickstead, R. and Furnham, A. (2017), "Comparing mental health literacy and physical health literacy: an exploratory study", Journal of Mental Health, Vol. 26 No. 5, pp. 449-456, doi: 10.1080/09638237.2017.1294743.

Wong, E.C., Collins, R.L. and Cerully, J.L. (2015), "Reviewing the evidence base for mental health first aid: is there support for its use with key target populations in California?", Rand Health Quarterly, Vol. 5 No. 1, pp. 1-9.

\section{Further reading}

Health Education England (2020a), Investing in People: Workforce Plan for England, HEE, London.

Health Education England (2020b), Final report. Leeds, available at: www.hee.nhs.uk/our-work/mental-wellbeing-report (accessed on 24 March 2020).

NHS England (2018), "Guidance on co-locating mental health therapists in primary care", NHS England, London, available at: www.england.nhs.uk/publication/guidance-on-colocating-mental-healththerapists-in-primary-care (accessed 23 March 2020).

\section{Acknowledgements}

This research received support from the author's institution's internal quality research (QR) fund ID number: 257.

\section{Corresponding author}

Andrew E.P. Mitchell can be contacted at: a.mitchell@chester.ac.uk

\section{About the author}

Dr Andrew E.P. Mitchell is based at the Department of Health and Social Care, University of Chester, Chester, UK. He is an Associate Professor of Mental Health at the University of Chester. His primary area of expertise is in mental health and psychological treatments. His current research studies include student nurses' psychological distress and service uptake, stress and coping behaviours, resilience and adaptive strategies. 
Table 1: Factor ranking for perceived stress scale and coping behaviour inventory

\begin{tabular}{|c|c|c|c|}
\hline Perceived Stress from & $\begin{array}{c}\text { Factor } \\
\text { ranking }\end{array}$ & M & SD \\
\hline Written assignments \& overall workload & 1 & 2.41 & 0.96 \\
\hline Low level of knowledge \& practice skills & 2 & 2.06 & 0.98 \\
\hline Peer group / everyday life & 3 & 1.77 & 0.89 \\
\hline The clinical environment & 4 & 1.70 & 1.02 \\
\hline Nursing care of patients & 5 & 1.66 & 0.76 \\
\hline Academic and clinical staff & 6 & 1.61 & 0.83 \\
\hline & & 1.84 & $\underline{0.68}$ \\
\hline
\end{tabular}

Coping strategy

\begin{tabular}{lllll}
\hline Problem-solving & 1 & 2.65 & 0.82 \\
Stay optimistic & & 2 & 2.32 & 0.70 \\
Transference & 3 & 2.18 & 0.89 \\
Avoidance & 4 & 0.66 & 0.56 \\
& $\underline{\text { Total CBI }}$ & & $\underline{1.88}$ & $\underline{0.48}$ \\
\hline
\end{tabular}

Note: $M$, mean; $S D$, standard deviation 
Table 2: Correlations between stress and coping variables

\begin{tabular}{lcccc} 
Perceived Stress from & $\begin{array}{c}\text { Avoidanc } \\
\mathrm{e}\end{array}$ & $\begin{array}{c}\text { Problem } \\
\text { solving }\end{array}$ & $\begin{array}{c}\text { Staying } \\
\text { optimistic }\end{array}$ & $\begin{array}{c}\text { Transferenc } \\
\mathrm{e}\end{array}$ \\
\hline Nursing care of patients & $.43^{* *}$ & .05 & .03 & .15 \\
Academic and clinical staff & $.54^{\star *}$ & .12 & -.06 & -.04 \\
Written assignments \& overall workload & $.50^{* *}$ & .07 & -.05 & .01 \\
Peer group / everyday life & $.52^{* *}$ & .15 & .01 & .11 \\
Low level of knowledge \& practice skills & $.40^{* *}$ & -.02 & .02 & .10 \\
The clinical environment & $.44^{* *}$ & -.03 & .01 & .12 \\
Total PPS & $.63^{* *}$ & .09 & -.01 & .12 \\
\hline
\end{tabular}

${ }^{\star \star}$ Correlation is significant at the .001 level (two-tailed). 
Table 3: Descriptive statistics for perceived stress scale and coping behaviors across year and field

\begin{tabular}{|c|c|c|c|c|c|c|c|}
\hline & $\mathrm{N}$ & $\mathrm{M}$ & S.D. & Skew & SE & Kurtosis & SE \\
\hline \multicolumn{8}{|l|}{ Perceived Stress Scale } \\
\hline Year 1 & 31 & 1.85 & .67 & -0.32 & 0.42 & -0.03 & 0.82 \\
\hline Year 2 & 29 & 1.79 & .78 & -0.42 & 0.43 & 0.42 & 0.85 \\
\hline Year 3 & 27 & 1.87 & .60 & -0.01 & 0.45 & -0.48 & 0.87 \\
\hline \multicolumn{8}{|c|}{ Coping Behaviour Inventory } \\
\hline Year 1 & 31 & 1.77 & .61 & -1.66 & 0.42 & 3.00 & 0.82 \\
\hline Year 2 & 29 & 2.04 & .39 & 0.53 & 0.43 & 0.50 & 0.85 \\
\hline \multirow[t]{2}{*}{ Year 3} & 27 & 1.83 & .35 & 0.37 & 0.45 & -1.10 & 0.87 \\
\hline & $\mathrm{N}$ & $\mathrm{M}$ & S.D. & Skew & SE & Kurtosis & SE \\
\hline \multicolumn{8}{|l|}{ Perceived Stress Scale } \\
\hline Adult & 60 & 1.84 & 0.68 & -0.29 & 0.31 & 0.45 & 0.61 \\
\hline Mental health & 15 & 1.72 & 0.70 & -0.25 & 0.58 & -0.30 & 1.12 \\
\hline Learning disabilities & 10 & 2.08 & 0.71 & -1.26 & 0.69 & 2.22 & 1.33 \\
\hline Child \# & 2 & 1.60 & 0.37 & - & - & - & - \\
\hline \multicolumn{8}{|c|}{ Coping Behaviour Inventory } \\
\hline Adult & 60 & 1.87 & 0.44 & -1.11 & 0.31 & 4.38 & 0.61 \\
\hline Mental health & 15 & 1.85 & 0.41 & -1.01 & 0.58 & 1.07 & 1.12 \\
\hline Learning disabilities & 10 & 2.12 & 0.45 & 0.54 & 0.69 & 0.35 & 1.33 \\
\hline Child \# & 2 & 0.97 & 1.38 & - & - & - & - \\
\hline
\end{tabular}

Note: $M$, mean; $N$, number; $S D$, standard deviation; SE, standard error; \# sample size is too small to calculate Skew and Kurtosis. A smaller critical value for rejecting the null hypothesis was utilised after dividing Skew or Kurtosis value by its standard error (Std. Error) and comparing it to a critical value of \pm 1.96 rather than \pm 2.58 (Rose, Spinks \& Canhoto, 2015). All values within critical threshold limit. 
Table 4: Linear multiple regression analyses for perceived stress and coping

behaviors $(n=87)$

\begin{tabular}{lccccc} 
Perceived stress & $\begin{array}{c}\text { Unstandardized } \\
\text { Coefficient } \\
\text { Beta (B) }\end{array}$ & $\mathrm{t}$ & $\mathrm{p}$ & $\mathrm{R}^{2}$ & Total \\
\hline Avoidant behaviour & 0.728 & 6.88 & $0.001^{* *}$ & 0.358 & $35.8 \%$ \\
Coping Behaviours & $\begin{array}{c}\text { Unstandardized } \\
\text { Coefficient } \\
\text { Beta (B) }\end{array}$ & $\mathrm{t}$ & $\mathrm{p}$ & $\mathrm{R}^{2}$ & Total \\
\hline Peer group / everyday life & 0.204 & 3.78 & $0.001^{* *}$ & 0.144 & $14.4 \%$
\end{tabular}

Note. ${ }^{*} p<.05,{ }^{* *} p<.001 ; B$, Unstandardized regression coefficient; $\mathrm{R}^{2}$, R squared; $t$ t-value; \%, total variance attributable to that variable alone 